\title{
Postnatal Developmental Expression Profile Classifies the Indusium Griseum as a Distinct Subfield of the Hippocampal Formation
}

\author{
Marie Sanders*, Elisabeth Petrasch-Parwez, Hans-Werner Habbes, Monika v. Düring * \\ and Eckart Förster*
}

Department of Neuroanatomy and Molecular Brain Research, Ruhr-University Bochum, Bochum, Germany

OPEN ACCESS

Edited by:

Igor Jakovcevski,

Helmholtz Association of German

Research Centers (HZ), Germany

Reviewed by:

Thomas Deller,

Goethe University Frankfurt, Germany Robert Francis Hevner, University of California, San Diego, United States

*Correspondence: Marie Sanders marie.sanders@rub.de

Monika v. Düring

monika.duering@rub.de Eckart Förster

eckart.foerster@rub.de

Specialty section: This article was submitted to Cell Adhesion and Migration, a section of the journa Frontiers in Cell and Developmental Biology

Received: 09 October 2020 Accepted: 07 December 2020 Published: 12 January 2021

Citation:

Sanders M, Petrasch-Parwez E, Habbes H-W, Düring Mv and Förster E (2021) Postnatal Developmental Expression Profile Classifies the Indusium Griseum as a Distinct Subfield of the Hippocampal Formation. Front. Cell Dev. Biol. 8:615571. doi: 10.3389/fcell.2020.615571
The indusium griseum (IG) is a cortical structure overlying the corpus callosum along its anterior-posterior extent. It has been classified either as a vestige of the hippocampus or as an extension of the dentate gyrus via the fasciola cinerea, but its attribution to a specific hippocampal subregion is still under debate. To specify the identity of IG neurons more precisely, we investigated the spatiotemporal expression of calbindin, secretagogin, Necab2, PCP4, and Prox1 in the postnatal mouse IG, fasciola cinerea, and hippocampus. We identified the calcium-binding protein Necab2 as a first reliable marker for the IG and fasciola cinerea throughout postnatal development into adulthood. In contrast, calbindin, secretagogin, and PCP4 were expressed each with a different individual time course during maturation, and at no time point, IG or fasciola cinerea principal neurons expressed Prox1, a transcription factor known to define dentate granule cell fate. Concordantly, in a transgenic mouse line expressing enhanced green fluorescent protein (eGFP) in dentate granule cells, neurons of IG and fasciola cinerea were eGFP-negative. Our findings preclude that IG neurons represent dentate granule cells, as earlier hypothesized, and strongly support the view that the IG is an own hippocampal subfield composed of a distinct neuronal population.

Keywords: indusium griseum, fasciola cinerea, dentate gyrus, CA2 region, Necab2, Prox1, secretagogin, PCP4

\section{INTRODUCTION}

The indusium griseum (IG) is a thin bilateral stripe-like allocortical brain area covering the entire anterior-posterior extent of the corpus callosum at the basis of the cingulate cortex. According to its topographical localization, the IG can be subdivided into an anterior part bending around the genu of the corpus callosum, a dorsal part overlying the corpus callosum surface, and a posterior part around the splenium of the corpus callosum, closely associated with the fasciola cinerea (FC) (Künzle, 2004). The long anterior-posterior expansion of this otherwise narrow structure implies proximity to several different brain areas. While there is consensus to consider the IG as part of the limbic system (Di Ieva et al., 2015), its allocation to a specific neuroanatomical structure is still under debate.

The IG was initially described as a rudimental continuation of the hippocampus remaining on the dorsal surface during relocation of the hippocampus to a more basal position during 
phylogenesis (Elliot Smith, 1897), a view that is still favored in more recent studies (Wyss and Sripanidkulchai, 1983; Adamek et al., 1984). In more detail, the IG has been considered either as a remnant of the subiculum, of the cornu ammonis or, in particular, of the dentate gyrus (DG) connected to it via the FC. The latter, most common view is mainly based on similarities between IG and DG concerning cytoarchitecture, cell morphology, and neuronal projections (Wyss and Sripanidkulchai, 1983; Adamek et al., 1984; Laplante et al., 2013). Finally, it has also been suggested that the IG should be considered as a distinct subregion within the hippocampal formation (Künzle, 2004). The idea that the IG is more than a mere hippocampal rudiment is supported by a recent histological and magnetic resonance imaging study on the human IG clearly confirming that it does not show any signs of regression during postnatal development (Bobic Rasonja et al., 2019). Of note, the IG has gained considerable attention because astrocytes derived from this region seem to play a key role as guidepost cells for the first pioneer fibers of the murine corpus callosum (Shu et al., 2003; Smith et al., 2006; Lavado et al., 2014; Di Ieva et al., 2015). Furthermore, IG neurons in the mouse brain expressing the transcription factor Rfx3 contribute to the formation of both the hippocampal fissure and the corpus callosum (Benadiba et al., 2012). Apart from this transient function as a source of guideposts, another role of the IG during development gets significant support by a recent study describing the IG as an important prenatal relay station in developing limbic and olfactory circuits (Fuzik et al., 2019). However, more specific functions of the mature IG remain elusive.

To clarify the ongoing controversial discussion on the identity of the IG, we analyzed the postnatal spatiotemporal expression pattern of calbindin, secretagogin, N-terminal EFhand calcium-binding protein 2 (Necab2), and of calmodulinbinding Purkinje cell protein 4 (PCP4) in the mouse IG, FC, and hippocampus. Finally, the previously hypothesized common identity of IG pyramidal and dentate granule cells was explored by investigating the protein expression of the transcription factor Prospero-related homeobox protein 1 (Prox1), known to define dentate granule cell fate, and by analyzing IG, FC, and DG neurons in transgenic mice that express enhanced green fluorescent protein (eGFP) in early postmitotic dentate granule cells (Overstreet et al., 2004).

\section{MATERIALS AND METHODS}

\section{Animals}

All mice were treated in accordance with the guidelines of the German animal protection law, maintained in a darklight cycle of $12 / 12 \mathrm{~h}$, and provided with food and water ad libitum. C57BL/6J wild-type mice were purchased from the Jackson Laboratory (Bar Harbor, ME, United States) and bred in the animal house of the Medical Faculty, Ruhr-University Bochum (Germany).

To cover broad developmental stages, we used two mice each at postnatal day (p) $0,5,15,20,25$, and 6 months of age for cresyl violet staining, as well as two mice each at $\mathrm{p} 0,2,5,8,10,12$,
15,20 , and 25 , and two adult mice each, aged 6 and 12 months for immunostaining.

To compare immature dentate granule cells with IG and FC neurons, brains of proopiomelanocortin (POMC)-eGFP transgenic mice (Overstreet et al., 2004), bred in the animal facility of the Center for Molecular Neurobiology Hamburg (ZMNH, University Medical Center Hamburg-Eppendorf, Germany), were examined at p0.

\section{Tissue Preparation}

Mice aged p0-p25 were deeply anesthetized by $\mathrm{CO}_{2}$ asphyxiation and decapitated. The brains were quickly removed, immersion fixed in $4 \%$ paraformaldehyde (PFA) in $0.1 \mathrm{M}$ phosphate buffer ( $\mathrm{pH} 7.4$ ) for 1 week, and then transferred to $0.5 \%$ PFA until further processed. Adult mice aged 6 and 12 months were deeply anesthetized with pentobarbital potassium (Narcoren ${ }^{\circledR}$, $500 \mathrm{mg} / \mathrm{kg}$, Merial GmbH, Germany) as described (PetraschParwez et al., 2007). Briefly, after opening the thorax, $0.3 \mathrm{~mL}$ Liquemin N 25000 (Roche, Basel, Switzerland) was intracardially injected followed by a transcardial perfusion with 4\% PFA for $20 \mathrm{~min}$. The brains were removed, post-fixed overnight in $4 \%$ PFA, and then transferred to $0.5 \%$ PFA in $0.1 \mathrm{M}$ phosphate buffer (pH 7.4) until used.

For peroxidase immunohistochemistry, all brains were cut into $40-\mu \mathrm{m}$-thick coronal vibratome sections starting anterior of the corpus callosum genu, ending occipital of the corpus callosum splenium and immunostained free floating as described below.

For immunofluorescence histochemistry, POMC-eGFP transgenic mice were decapitated at p0. Entire brains were immersion-fixed in 4\% PFA for 1 week, washed in $0.1 \mathrm{M}$ phosphate buffer, cut into $50-\mu \mathrm{m}$ vibratome sections, mounted on Superfrost Slides, air-dried for $30 \mathrm{~min}$, and subsequently stained as described below.

\section{Peroxidase Immunohistochemistry}

Sections were immunostained with primary antibodies in a repeated order as previously described (Petrasch-Parwez et al., 2007). Every 10th section of the brains was stained with primary antibodies against calbindin (Swant, Marly, Switzerland; Cat\# CB 38, RRID: AB10000340; dilution: 1:20,000), secretagogin (Sigma-Aldrich, Munich, Germany; Cat\# HPA006641, RRID: AB1079874; dilution: 1:2,000), Necab2 (Sigma-Aldrich, Munich, Germany; Cat\# HPA013998, RRID: AB1848016; dilution: 1:20,000), PCP4 (Sigma-Aldrich, Munich, Germany; Cat\# HPA005792, RRID: AB1855086; dilution: 1:5,000), and Prox1 (Millipore, Billerica, United States; Cat\# AB5475, RRID: AB177485; dilution: 1:5,000).

Peroxidase immunohistochemistry was performed as previously described (Petrasch-Parwez et al., 2007). Briefly, the sections were reduced in $1 \% \mathrm{NaBH}_{4}$ in phosphate-buffered saline (PBS) for $30 \mathrm{~min}$, blocked in 10\% normal goat serum (NGS, Interchem, Pfaffen-Schwabenheim, Germany) in PBS with $0.3 \%$ Triton X-100 (SERVA Electrophoresis GmbH, Heidelberg, Germany), and then incubated with the respective primary antibody diluted in the blocking solution for $72 \mathrm{~h}$ at $4^{\circ} \mathrm{C}$. Afterward, the sections were rinsed in PBS, preincubated in $0.1 \%$ bovine serum albumin (BSA, 11930, SERVA 
Electrophoresis $\mathrm{GmbH}$, Heidelberg, Germany) in PBS for $30 \mathrm{~min}$, and incubated with a biotinylated goat anti-rabbit antibody (Vector Laboratories, Burlingame, CA, United States) overnight at $4^{\circ} \mathrm{C}$. Next day, the sections were again rinsed in PBS, incubated in $0.1 \%$ BSA in PBS, and treated with an avidin-biotinylated peroxidase complex (Vector Laboratories) for $4 \mathrm{~h}$ at room temperature. Peroxidase was visualized with 3,3'-diaminobenzidine. Finally, all sections were mounted on Superfrost Plus slides, air-dried for an hour, quickly dehydrated, and coverslipped.

\section{Cresyl Violet Staining}

Every 10th section was stained with $0.5 \%$ cresyl violet solution ( $\mathrm{pH}$ 4.8, Certistain ${ }^{\circledR}$, Art. Nr. 1.05235, Merck KGaA, Darmstadt, Germany) for morphological reference of adjacent immunostained sections.

Photodocumentation of cresyl violet and peroxidaseimmunostained sections was performed with an Olympus Microscope BH-2 equipped with a camera Olympus DP-71 (Olympus, Japan) and the computer-assisted software analysis Cell A (Soft imaging system $\mathrm{GmbH}$, Germany). The images were photoprocessed as TIFF files using Adobe Photoshop (version 2015) to adjust contrast and brightness.

\section{Immunofluorescence Histochemistry}

The mounted slides were rinsed twice in PBS, preincubated in $10 \%$ NGS for $30 \mathrm{~min}$, and incubated with the antibody against Necab2 (1:1,000), diluted in the previous solution for $24 \mathrm{~h}$. Then, slices were washed in PBS, preincubated with a $0.2 \%$ BSA/PBS solution for $30 \mathrm{~min}$, and incubated with a red-fluorescently labeled secondary antibody (Alexa Fluor ${ }^{\circledR}$ 633, Thermo Fisher Scientific Inc., Waltham, United States; RRID: AB2535732; $1: 1,000)$ in $0.2 \%$ BSA/PBS solution. After final washing in PBS, the slides were coverslipped in anti-fade medium (Prolog Gold, Molecular Probes ${ }^{\circledR}$, Thermo Fisher Scientific Inc., Waltham, United States). The immunofluorescence staining was imaged with an inverted Confocal Spinning-Disc-Laser Microscope (VisiScopeConfocal-Cell Explorer, Visitron Systems GmbH, Puchheim, Germany) and the computer-assisted software cellSens Entry (Olympus Life Science Solutions, Hamburg, Germany) and photoprocessed as described above.

\section{RESULTS}

\section{Cytoarchitecture of the IG and FC}

Cresyl violet staining of serial brain sections was performed to determine the spatial extension of the IG and FC subdivisions during development (Figure 1). These sections were also used as a morphological reference for immunostained sections. The IG and FC were identified according to the mouse brain atlases by Paxinos and Watson (2009) and Paxinos and Franklin (2019). At all postnatal stages, the subdivision of the IG into an anterior (Figures 1A,D,G,J,M), dorsal (Figures 1B,E,H,K,N), and posterior part was confirmed (Figures $\mathbf{1 C}, \mathbf{F}, \mathbf{I}, \mathbf{L}, \mathbf{O}$ ). The demarcation of the IG from the overlying cingulate cortex was already visible at p0 (Figures 1A,B). We detected a transition zone between IG and FC (Figure 1F), not observed between the IG and DG, which were always separated through the hippocampal fissure (Figure 1C). Of note, the FC directly neighbors the CA1 region, the medial part of $\mathrm{CA} 2$, ormore posteriorly-the subiculum (Figures 1C,F,I,L,O). The FC can be recognized by its pyramidal cell layer, which is oriented perpendicular to the hippocampal pyramidal cell layer (Figures 1I,O). The neuronal cell density of the IG decreased from anterior to posterior part in adult mice (Figures 1G-I,M$\mathbf{O})$. While the anterior IG is clearly separated by the fissura longitudinalis cerebri (Figures 1A,D,G,J,M), the dorsal IG partly merges with its contralateral counterpart (Figures 1B,E,H,K,N). The posterior IG is again divided into two separate parts by the fissura longitudinalis cerebri (Figures 1C,F).

\section{Calbindin Expression Decreases During Postnatal IG and FC Development}

Calbindin is known as a marker to distinguish hippocampal subdivisions (Celio, 1990; Kjonigsen et al., 2011). Therefore, we investigated its spatiotemporal distribution in the IG and FC compared to the hippocampus and DG (Figure 2). At p0, calbindin was strongly expressed in the anterior, dorsal, and posterior part of the IG and in the FC (Figures 2A-C). Calbindin-positive neurons accumulated particularly adjacent to IG pyramidal cells, which mainly lacked calbindin staining (Figures 2A,B,F,J,M,N). During postnatal development, calbindin expression gradually decreased in all subdivisions (Figures 2A-C,E-G,I-K,M-O). In the adult IG, only a few calbindin-positive neurons were detected within a faintly stained neuropil (Figures 2Qa-S). In detail, at p0, the IG contained numerous, intensely immunostained neurons at the border between the predominantly immunonegative IG pyramidal cell layer and the IG molecular layer, and few positive neurons that were scattered in the molecular and polymorph layer. At p5, calbindin-positive neurons were distributed in all three layers (Figure 2F) and were closely neighboring immunonegative pyramidal cells (Figures 2E,F). From stage p10 on, the density of calbindin-positive cells in the IG continuously decreased to few scattered neurons from p15 on into adulthood (Figures 2J,N,R). Beaded axons of calbindin-positive cells projected to the adjacent cortical areas, the corpus callosum, and to the contralateral hemisphere (Supplementary Figure S1A). As already detected in the IG, the FC also contained a large number of calbindin-immunopositive neurons at p0 (Figure 2C), which subsequently decreased during postnatal development (Figures 2G,K,O,S). Notably, calbindin immunostaining at p0 confirmed the conjunction of IG and FC (Figures 2C,D) as shown by cresyl violet staining. In the hippocampus proper and in the DG, calbindin immunostaining increased during postnatal development (Figures 2D,H,L,P,T). While positive cells were mainly found in the outer dentate granule cell layer during the first postnatal weeks, the subgranular zone of the DG in contrast, known to harbor neuronal stem cells, was immunonegative (Figures 2D,H,L,P). Almost all dentate granule cells were calbindin-positive in the adult mice (Figure 2T). Altogether, our investigations show that calbindin is a suitable marker for the IG and FC during early postnatal development, but not in 


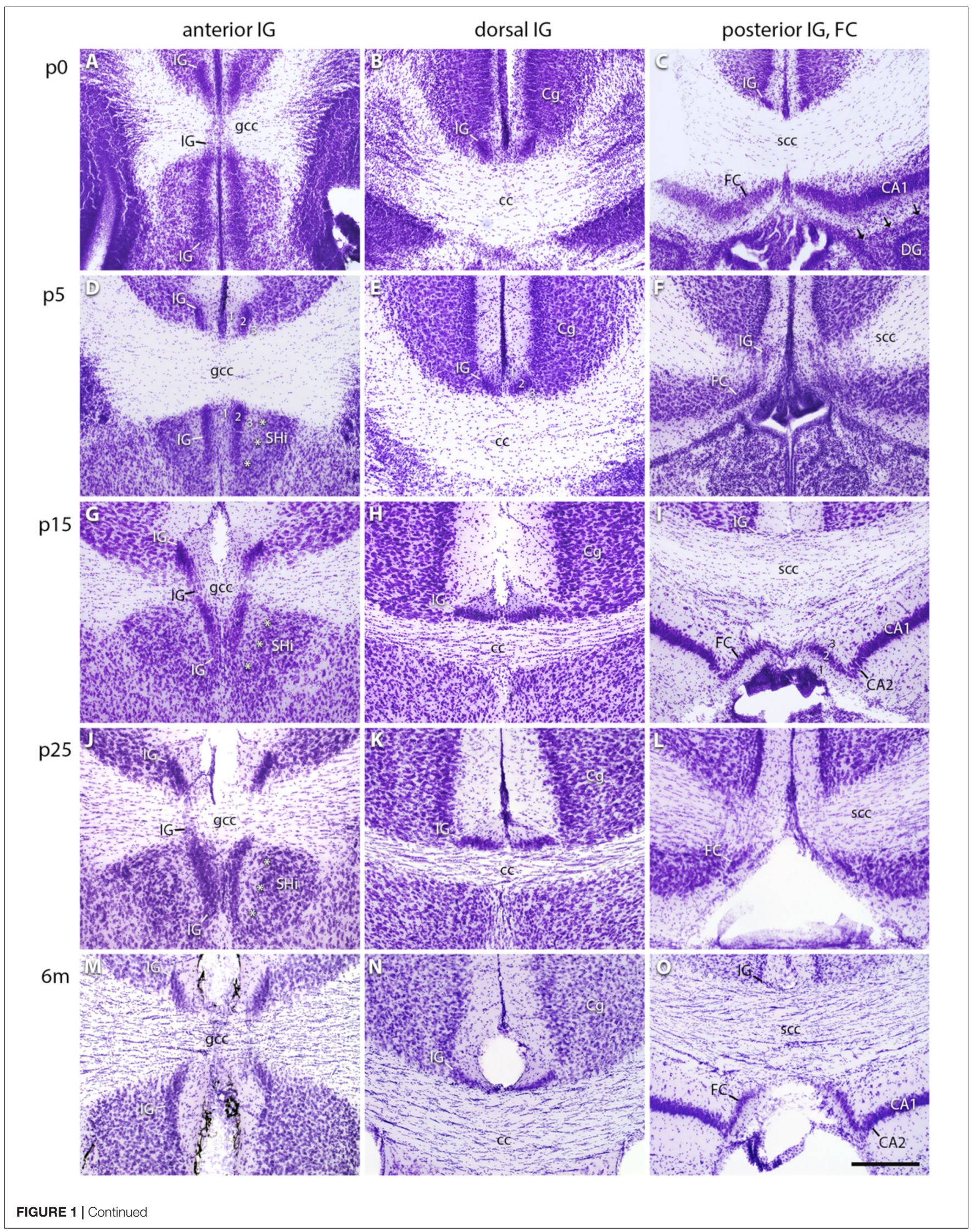


FIGURE 1 | Cytoarchitecture of the indusium griseum (IG) subdivisions. Cresyl violet-stained coronal sections of IG and FC in the mouse brain at p0 (A-C), p5 (D-F), p15 (G-I), p25 (J-L), and 6 months (m) of age (M-O) showing the anterior (A,D,G,J,M), dorsal (B,E,H,K,N), and posterior IG, the latter closely associated with the FC (C, F,I,L,O). Layers of the IG (1, 2, 3 in $\mathbf{D}, \mathbf{E}, \mathbf{I})$ differentiate during maturation, i.e., the IGs pyramidal cell layer (2, in $\mathbf{D}, \mathbf{E})$ flattens, and the polymorph layer $(\mathbf{D}, \mathbf{E})$ narrows with maturation, while the morphological adaptation of the molecular layer is less remarkable $\mathbf{( B , E , H , K , N )}$. Note the bending of the IG around the genu of corpus callosum (gcc, in $\mathbf{A}, \mathbf{G}, \mathbf{J}, \mathbf{M}$ ) and the transition of IG and FC around the splenium of corpus callosum (scc, in $\mathbf{F , L}$ ). The IG is neighboring the cingulate

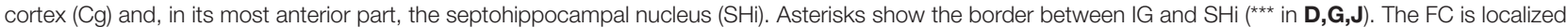
adjacent to the cornu ammonis (CA)1 (C) or to the medial CA2 region (I,O), but separated from the dentate gyrus (DG) through the hippocampal fissure (arrows in C). Differences in section level are due to tilted angles of section series. Scale bar $=200 \mu \mathrm{m}$ in (0) for (A-O).

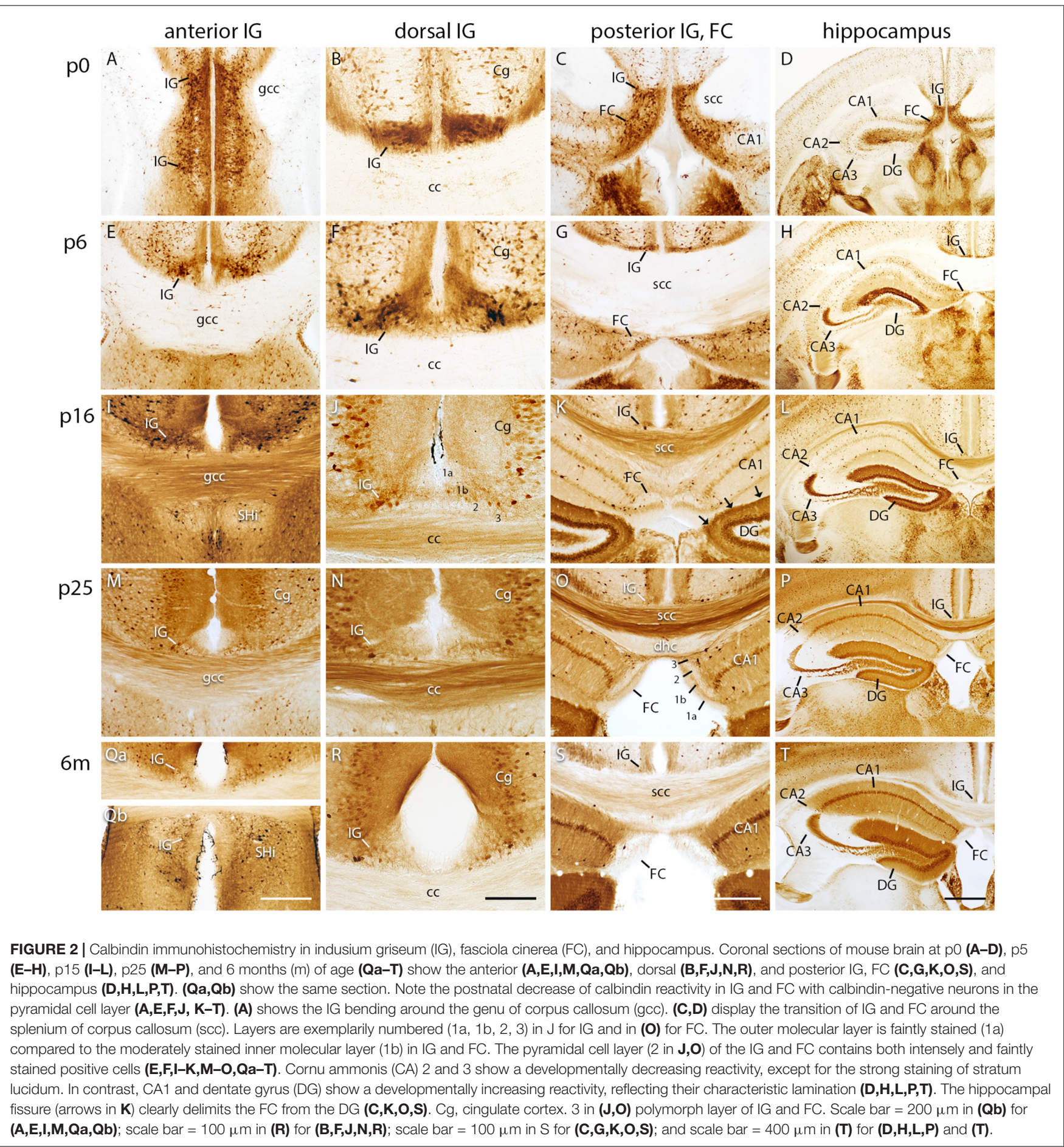




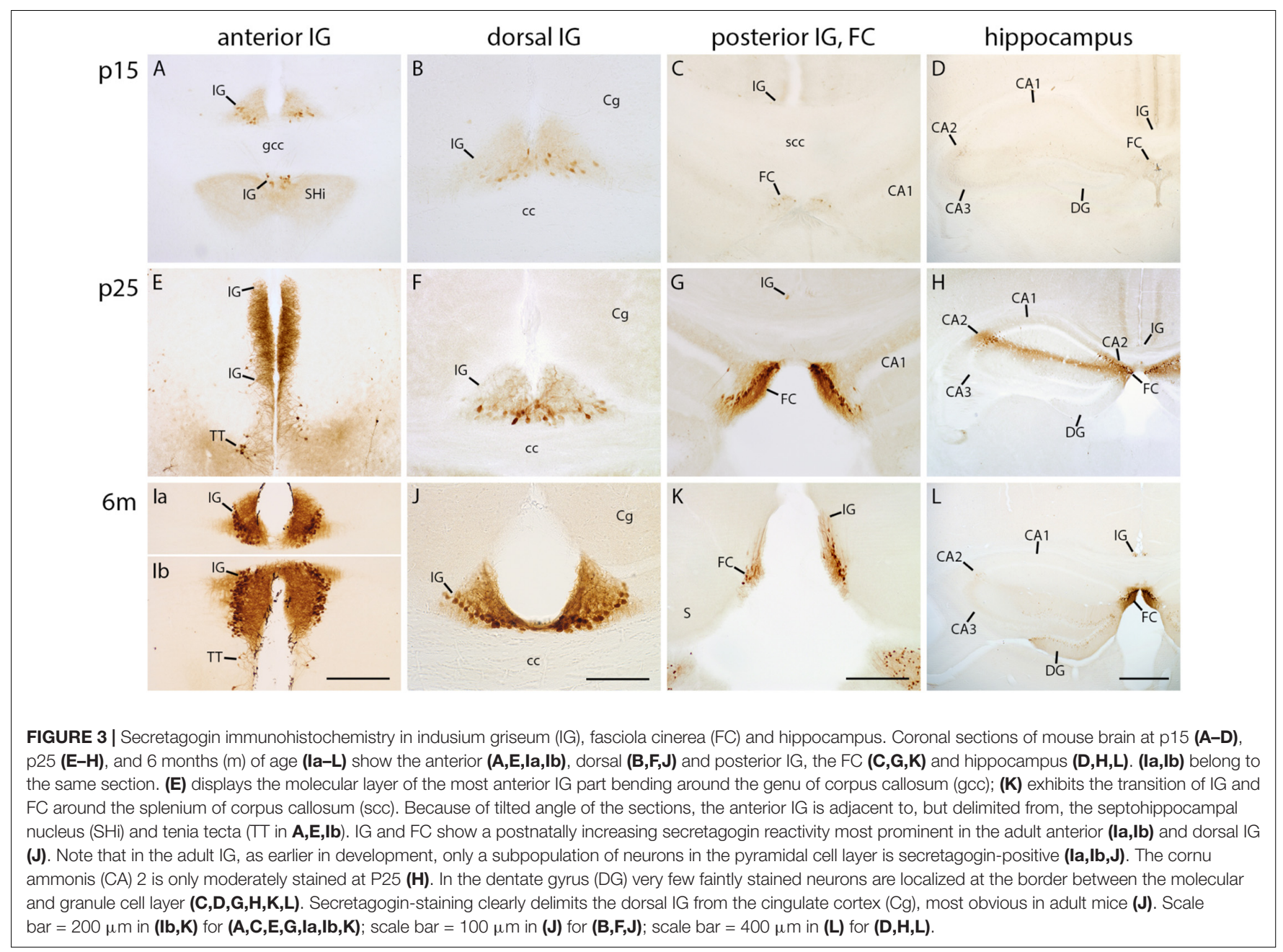

adult mice, much in contrast to the calbindin expression in the hippocampus proper and DG.

\section{Secretagogin Emerges Late During Postnatal IG and FC Development}

Secretagogin has previously been described as a marker for the IG in adult mice (Mulder et al., 2009). Therefore, we wanted to explore its developmental expression in the IG and FC. At p0, both the IG and FC lacked secretagogin reactivity (data not shown). At p5, only few faintly stained secretagogin-positive neurons were detected in the IG. Their quantity and staining intensity started to increase from the second postnatal week on, progressing from anterior to posterior (Figures $3 \mathrm{~A}-\mathrm{C}$ ). In the anterior IG, positive cells were predominantly densely arranged within the pyramidal cell layer (Figures $\mathbf{3 A}, \mathbf{E}, \mathbf{I a}, \mathbf{I b}$ ), whereas the dorsal part showed many immunopositive, but also unstained cells (Figures 3B,F,J). The majority of secretagoginpositive dendrites were oriented towards the molecular layer; some dendrites traversed to the contralateral IG (Supplementary Figure S1B). The heterogeneity, laminar distribution, and developmental expression pattern of secretagogin-positive cells in the FC were similar to those of the anterior IG (Figures
3C,G,K). The hippocampal CA2 region transiently showed a similar staining pattern as the IG from p15 to p25, with many secretagogin-positive pyramidal cells and an immunopositive stratum oriens and radiatum (Figures 3D,H). Later, in turn, the adult hippocampal CA2 region almost lacked secretagogin staining (Figure 3L). Faintly stained secretagogin-positive neurons appeared at the border of the dentate granule cell layer and the inner molecular layer in adult mice DG (Figure 3L). Our findings show that secretagogin is a useful marker for the late postnatal and mature IG and FC, but not for the early postnatal IG and FC. Finally, secretagogin expression of the hippocampal CA2 region was restricted to a limited time window during postnatal development.

\section{Necab2 Is a Novel Marker for IG and FC}

Necab2 has been shown to be a valuable marker for hippocampal subdivisions (Zimmermann et al., 2013). We therefore, investigated the developmental Necab2 protein expression in the IG and adjacent structures. Interestingly, Necab2 immunostaining in the IG and FC was already prominent at p0 (Figures 4A-C). The intense immunostaining was consistent through all postnatal stages along the entire anterior-posterior 


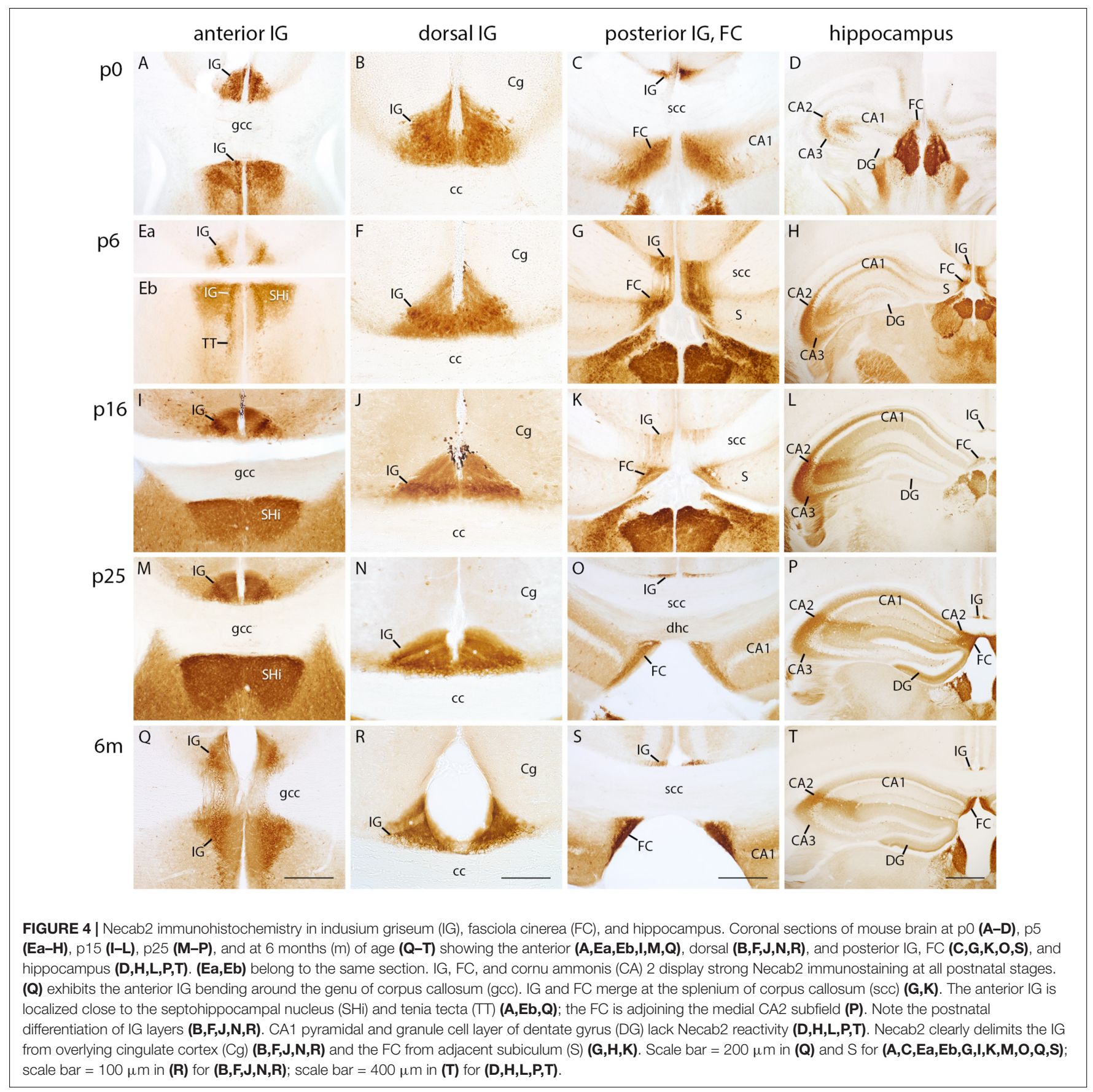

extent of the IG and FC (Figure 4), thereby designating Necab2 as a novel marker for these structures. In more detail, both, IG and FC, revealed a trilaminar staining pattern with a strong immunoreactivity in all layers. The pyramidal cell layer exhibited many immunopositive and scattered immunonegative neurons (Figures 4A-C,E-G,I-K,M-O,Q-S). Necab2 staining also confirmed the conjunction between IG and FC (Figure 4G). Importantly, the dentate granule cell layer was devoid of Necab2 staining at all investigated stages, whereas the hippocampal CA2 region displayed Necab2-staining throughout postnatal development into adulthood, although slightly delayed (starting by p5) when compared to the IG (Figures $4 \mathrm{D}, \mathbf{H}, \mathbf{L}, \mathbf{P}, \mathbf{T}$ ). In conclusion, we show that Necab2 is a reliable marker for the IG and FC throughout all postnatal stages, in contrast to the immunonegative dentate granule cell layer, which was devoid of Necab2.

\section{PCP4 Expression Decreases in the IG During Postnatal Development}

As Necab2 and secretagogin immunostaining pointed to similarities between IG, FC, and CA2, we studied the expression 


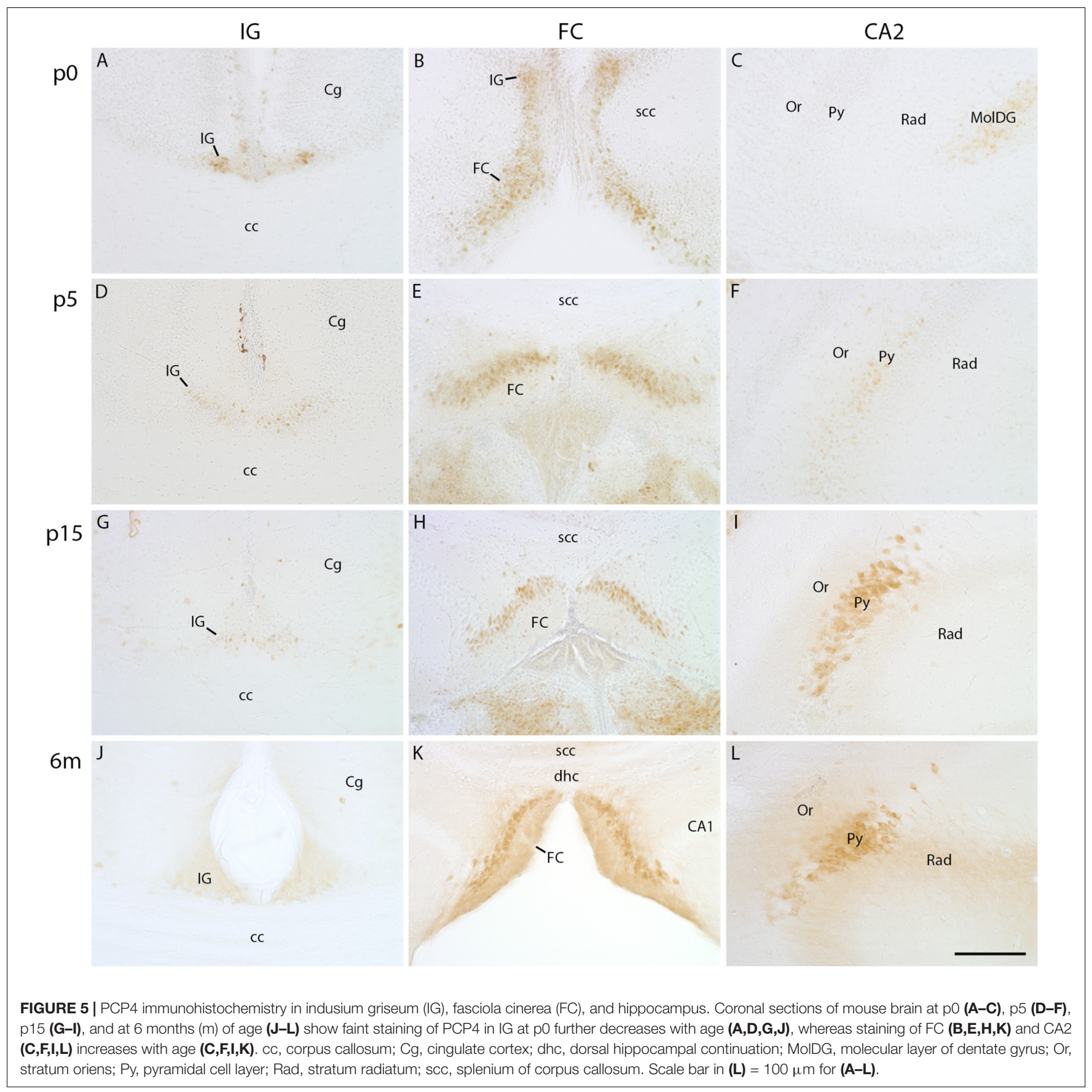

of PCP4, a specific marker for CA2 pyramidal cells (Lein et al., 2005; Laeremans et al., 2013; San Antonio et al., 2014). At p0, faint PCP4 immunostaining was visible in the IG and FC with a continuous transition of PCP4-positive cells from IG to FC (Figure 5B), whereas CA2 lacked PCP4 at that stage (Figure 5C). During postnatal development, the intensity of initially PCP4-positive IG cells (Figure 5A) declined, and later the IG was devoid of PCP4 immunoreactivity (Figures 5D,G,J). In contrast, the pyramidal cell layer of the FC (Figures 5B,E,H,K) contained PCP4-positive neurons at all postnatal stages (Figures $\mathbf{5 B}, \mathbf{E}, \mathbf{H}, \mathbf{K}$ ) and the CA2 region from around $\mathrm{p} 5$ on (Figures 5C,F,I,L), both with increasing intensity. Altogether, PCP4 immunostaining revealed characteristic spatiotemporal differences in the investigated subregions, i.e., a developmental decrease of IG and an increase of PCP4-positive FC and CA2 neurons.

\section{IG and FC Neurons Do Not Express the Dentate Granule Cell Marker Prox1}

Previous studies described a similar morphology of IG pyramidal and dentate granule cells (Adamek et al., 1984). Prox1, 


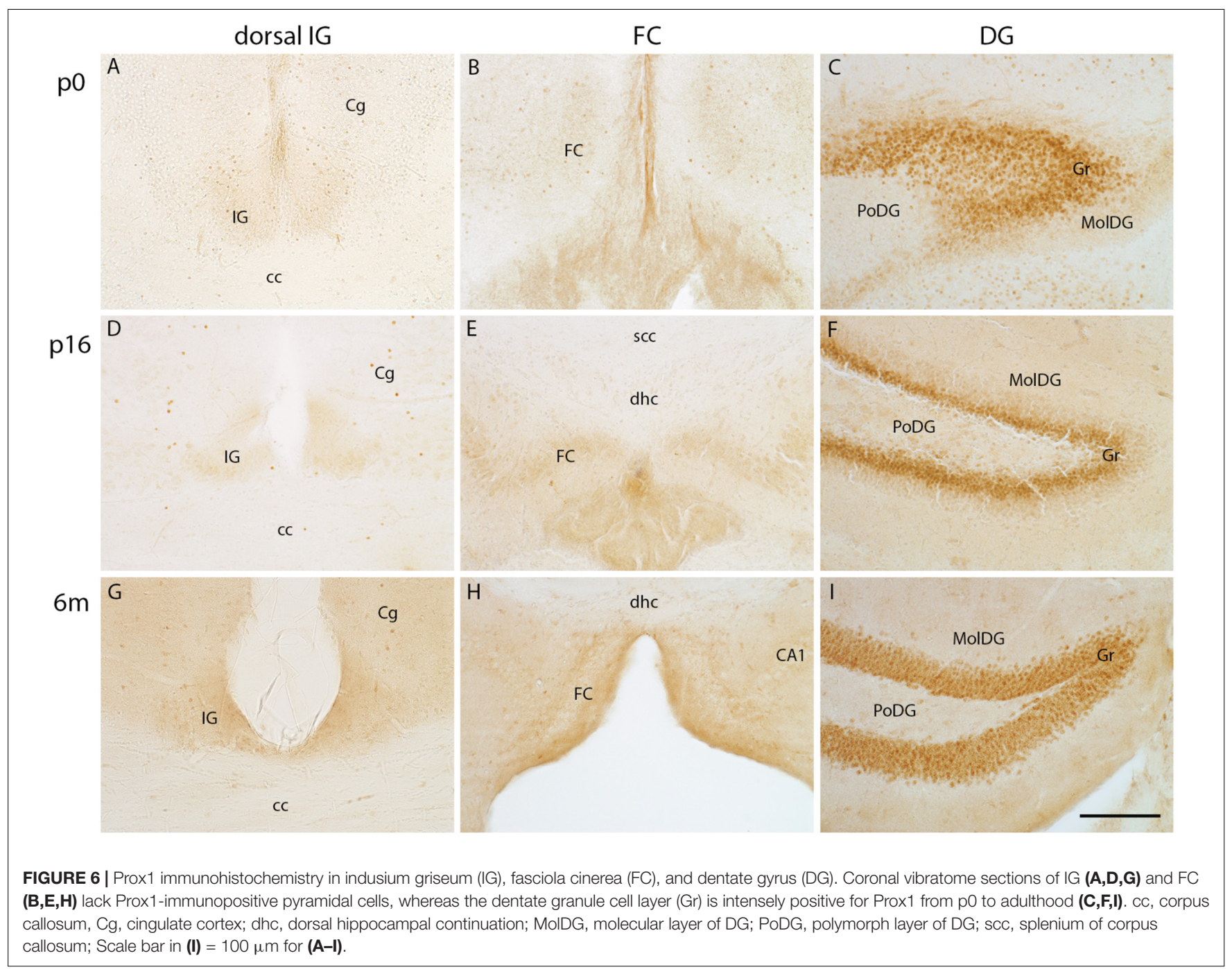

a transcription factor that is expressed in dentate granule cells throughout embryonic and postnatal development into adulthood, is known to define dentate granule cell fate (Lavado and Oliver, 2007; Iwano et al., 2012). Here, we investigated Prox1 protein expression in the IG and FC from early postnatal stages to adulthood. The Proxl antibody immunostained nuclei of dentate granule cells at all postnatal stages (Figures 6C,F,I), most intensely in the subgranular zone (Figure 6F) adjacent to the polymorph layer of the DG. In contrast, Prox1 immunostaining was absent in the IG (Figures $\mathbf{6 A}, \mathbf{D}, \mathbf{G}$ ) and FC (Figures 6B,E,H) at all stages investigated. In conclusion, Prox1 immunostaining clearly distinguished IG and FC neurons from dentate granule cells.

\section{eGFP Expression in POMC-eGFP Mice Discerns IG and FC Neurons From Dentate Granule Cells}

Finally, we analyzed brain sections of p0 transgenic mice expressing eGFP under control of POMC promotor sequences. In this mutant, newly generated postmitotic dentate granule cells transiently express eGFP for approximately 4 weeks (Overstreet et al., 2004). Fluorescence microscopy confirmed that dentate granule cells expressed eGFP. In contrast, in the IG, the FC and the hippocampal pyramidal cell layer eGFP expression was absent (Figure 7). Vice versa, fluorescent immunostaining with the Necab2 antibody labeled IG and FC neurons, but not dentate granule cells (Figures 7A,B). Necab2 immunofluorescence also confirmed that IG and FC form a continuum toward the hippocampal CA2 region (Figure 7B). Thus, combined eGFP fluorescence and Necab2 immunofluorescence clearly distinguished IG and FC neurons from dentate granule cells.

\section{DISCUSSION}

In the present study, we characterized the developmental expression pattern of the calcium-binding proteins calbindin, secretagogin, and Necab2, the calmodulin-binding protein PCP4, the transcription factor Proxl, and of eGFP under control of the POMC promotor in transgenic mice, in the IG, FC, and the hippocampus. We found that Necab2 is strongly 


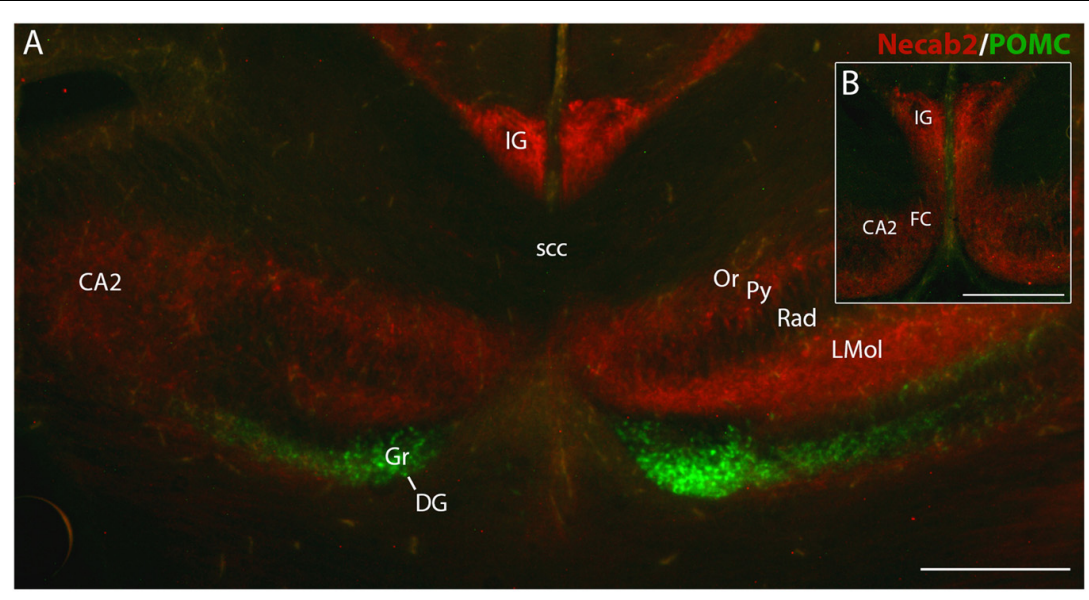

FIGURE 7 | Necab2 immunofluorescence in indusium griseum (IG), fasciola cinerea (FC), and hippocampus in a POMC-eGFP transgenic mouse brain at p0. Adjacent coronal sections show non-overlapping expression of POMC-eGFP (green) and Necab2 immunofluorescence (red). POMC-eGFP is localized in granule cells (Gr) of dentate gyrus (DG). IG, FC, and cornu ammonis (CA) are Necab2-immunopositive (A,B). Note the close topographical association of IG, FC, and medial CA2 (B). Necab2 appears more intense in IG than in CA2 and FC (B). It is also detected in the lacunosum molecular layer (LMol) of CA, in the stratum oriens (Or) and pyramidal cell layer (Py) of CA2. CA1 shows faint immunofluorescence in Or, Py, and stratum radiatum (Rad). scc, splenium of corpus callosum. Scale bar in (B) $=20 \mu \mathrm{m}$ for $(\mathbf{A}, \mathbf{B})$.

expressed in the IG and FC during all postnatal stages until adulthood, identifying Necab2 as a novel reliable marker for the mouse IG and FC. In addition, the IG forms a continuous Necab2-immunopositive structure together with the FC, closely neighboring the medial portion of the hippocampal CA2 region. In contrast, calbindin, secretagogin, and PCP4 are only transiently expressed in the IG and FC.

Remarkably, IG neurons did not express Prox1, a transcription factor known to define granule cell fate, thereby disproving the often-stated hypothesis that IG neurons have the same developmental fate as dentate granule cells. Moreover, our finding that IG and FC neurons, much in contrast to dentate granule cells, did not express eGFP under control of the POMC promotor in a transgenic mouse model corroborates this interpretation. Our immmunohistochemical data on calbindin, Necab2, and Prox1 are bolstered by in situ hybridization data for the RNA encoding these proteins (ㄷ) 2015 Allen Institute for Brain Science. Allen Brain Atlas API. Available from: Calbindin: https://mouse.brain-map.org/experiment/ thumbnails/79556672?image_type=ish\&popup=true;

Necab2: http://developingmouse.brain-map.org/experiment/ thumbnails/73788010?image_type=ish\&popup=true; Prox1: http://mouse.brain-map.org/experiment/thumbnails/73520980? image_type=ish\&popup=true). Altogether, our findings strongly support the view that the IG should be addressed as a distinct subregion of the hippocampal formation.

\section{Calcium- and Calmodulin-Binding Proteins Characterize IG and FC}

Calcium-binding proteins are important for the regulation of intracellular calcium homeostasis and transport. The here examined expression patterns of the calcium-binding proteins calbindin, secretagogin, and Necab2 have been described as mostly non-overlapping, making them useful markers to identify selective brain areas and specific neuronal subpopulations (Celio, 1990; Jacobowitz and Winsky, 1991; Mulder et al., 2009; Zimmermann et al., 2013). Mulder et al. (2009) demarcated the calbindin-positive cingulate cortex from the calbindinnegative IG. Our findings, however, reveal that calbindin is temporally strongly expressed in the IG, i.e., during early postnatal development, decreasing continuously until adulthood. In addition, the here observed spatiotemporal expression of calbindin clearly differs between IG and FC compared to the DG, as the expression of calbindin increases during maturation of dentate granule cells.

Secretagogin is expressed in neurons of the mammalian corticolimbic system (Mulder et al., 2009); immunopositive cells were shown to be positioned along the FC, the IG, and anterior hippocampal continuation. As IG neurons, i.e., secretagoginpositive neurons, morphologically and physiologically resemble dentate granule cells (Wyss and Sripanidkulchai, 1983; Adamek et al., 1984; Mulder et al., 2009), a common developmental origin of dentate granule cells and IG pyramidal cells has been proposed. This hypothesis has recently been questioned by Fuzik et al. (2019), who detected that the IG neurons lack Prox1-expression in young adult mice. This observation was confirmed in our study and even substantially extended, as we included newborn mice in our investigation on the IG and FC, both of which were also devoid of Prox1 immunoreactivity. The presence of scattered secretagogin-positive cells at the border between dentate granule cell and inner molecular layer (Mulder et al., 2009) was also confirmed in our study.

Secretagogin-positive cells are often found in vicinity to the ventricular system with dendrites oriented along the pial surface, which might display a role in releasing neuroactive substances into the cerebrospinal fluid (Mulder et al., 2009, 2010). Further assumed functions are a neuroendocrine role in 
vesicle exocytosis (Bauer et al., 2011), microtubules dynamics (Maj et al., 2010, 2012), and a possible neuroprotective role against the neurodegeneration as in Alzheimer disease (Attems et al., 2008). Altered secretagogin expression has been described to possibly reflect cellular dysfunction of locus coeruleus neurons in Alzheimer disease (Zahola et al., 2019). Moreover, secretagogin may be involved in neuronal differentiation persisting during neurogenesis until adulthood (Alpár et al., 2012). In this regard, it would be of great interest to further examine the function of secretagogin-positive cells within the IG and FC.

Fuzik et al. (2019) detected calbindin D28k RNA within secretagogin-positive excitatory neurons while we observed a mainly non-overlapping time course of calbindin and secretagogin protein expression within the IG and FC. Our finding that both calbindin expression and secretagogin expression within the IG and FC have a characteristic time course during postnatal development reflects a particular differentiation of IG neurons and argues against the view that the IG might represent a mere hippocampal rudiment, as stated earlier (Elliot Smith, 1897; Macchi, 1951; Di Ieva et al., 2015). Instead, the characteristic postnatal time course of calciumbinding protein expression argues in favor of a functional role of the IG beyond its transient role as a source of guidepost cells in mice, required for the formation of the murine corpus callosum (Shu et al., 2003; Smith et al., 2006; Lavado et al., 2014; Di Ieva et al., 2015). This assumption is in line not only with the results of Fuzik et al. (2019) as mentioned above, but also with recent findings revealing a continuous maturation of the fetal human IG (Bobić Rasonja et al., 2019) and clearly excluding its postnatal regression.

In our study, we identified Necab2 as a novel reliable marker protein for the anterior, dorsal, and posterior IG and also for the FC throughout all postnatal stages, clearly demarcating these brain areas from adjacent structures. The strong and selective Necab2 reactivity will facilitate future studies on the IG and FC, as these narrow structures are difficult to delimit especially in their anterior and posterior parts. It would be of great interest to investigate Necab2 in the respective brain areas of other mammalian species including humans. Necab1, Necab2, and Necab3 are evolutionary highly conserved homolog calciumbinding proteins that were originally described as targets of the calcium sensorsynaptotagmin I and of the transcription factor Pax6, which is an important regulatory gene for proper eye and brain development (Bernier et al., 2001; Sugita et al., 2002). Interestingly, Necab2 has been identified as a pronociceptive molecular determinant of inflammatory pain within dorsal root ganglia and spinal cord interneurons (Zhang et al., 2018). Its downregulation prevented persistent pain and facilitated functional recovery (Zhang et al., 2018).

PCP4 has been described as a specific marker for the hippocampal CA2 region from around $\mathrm{P} 4$ on, for the CA3 region, and the dentate granule cell layer (Lein et al., 2005; San Antonio et al., 2014). The medial CA2 region and the FC form a PCP4-positive continuum as we confirm in our study, even though neurons of these two regions are not identical (Lein et al., 2005). PCP4 is known to play an important role concerning neuroendocrine cell differentiation and release of neurotransmitters (Ziai et al., 1986, 1988; Harashima et al., 2011; Renelt et al., 2014) and as an inhibiting modulator of $\mathrm{Ca}^{2+}$ calmodulin signaling controlling the resistance of neurons to toxicity (Recabarren and Alarcón, 2017). Dysregulated PCP4 expression was described in the context of neuronal disorders such as Alzheimer disease (Slemmon et al., 1994; Recabarren and Alarcón, 2017), Huntington disease (Utal et al., 1998), major depressive disorder (Teyssier et al., 2011), and Down syndrome (Cabin et al., 1996; Chen et al., 1996) and related to alterations in the prefrontal cortex of individuals suffering from alcohol abuse (Iwamoto et al., 2004). The human IG has been reported to be more resistant to factors triggering histological changes occurring in the aged and Alzheimer diseases hippocampus, i.e., to the production of abnormal proteins associated with Alzheimer disease (Lippa et al., 1990; Lippa and Smith, 1992). Considering the fact that the CA2 region of the hippocampus also belongs to the brain regions that have been described as more resistant to degeneration and the occurrence of neurofibrillary tangles (Ishizawa et al., 2002), further studies on the apparently partially overlapping protein expression pattern of IG, FC, and CA2 could be of great interest. Notably, our findings revealed spatiotemporal differences in PCP4 expression concerning the IG, FC, and CA2. It would be interesting to expand the comparison between IG, FC, and CA2 by using additional markers that may elucidate functional differences of these areas.

\section{Prox1 and POMC Promoter-Driven eGFP Expression in Dentate Granule Cells}

Prox1 has first been cloned and described by Oliver et al. (1993) as a mouse homolog of the Drosophila homeobox gene prospero, playing a role in early development of the central nervous system. It has been identified as the transcription factor that defines dentate granule cell identity, and it regulates cell proliferation in the dentate neuroepithelium during early development (Lavado et al., 2010; Iwano et al., 2012). If Prox1 is missing post-mitotically in immature dentate granule cells, they differentiate into neurons with the characteristic morphology and gene expression pattern of CA3 pyramidal cells (Iwano et al., 2012). In the absence of Prox1, intermediate progenitor cells are reduced and dentate granule cells cannot terminally differentiate (Lavado et al., 2010). As revealed by Fuzik et al. (2019), excitatory IG neurons lack Prox1 mRNA. We expanded this finding by studying the protein expression of Prox1 in the IG and FC during postnatal development. Our results revealed that IG pyramidal cells do not express Prox1 at any postnatal stage, precluding the same fate of dentate granule cells and IG neurons.

To corroborate our interpretation that IG neurons have a different developmental fate than dentate granule cells, we took advantage of a transgenic mouse model expressing eGFP under the transcriptional control of POMC genomic sequences. eGFP expression is also seen in adult-born dentate granule cells that are generated by adult stem cells. Therefore, absence of eGFP expression in the IG and FC in this transgenic mouse model excludes that these neurons have the developmental fate of dentate granule cells, despite their dentate granule celllike morphology. 


\section{The IG as a Distinct Subregion of the Hippocampal Formation}

There has been an ongoing debate on the classification of the IG, as morphological studies so far did not allow a clear assignment to any distinct brain structure. Künzle (2004) divided the IG of the hedgehoc tenrec into an anterior precallosal, dorsal supracallosal, and posterior postcallosal portion. This subdivision is also confirmed in our study in the mouse IG. While the term indusium griseum is generally used in the neuroanatomical nomenclature to designate all three parts together (Paxinos and Watson, 2009), the precallosal part was also considered separately and referred to as the so-called anterior hippocampal continuation (Wyss and Sripanidkulchai, 1983). The latter term has also been used to combine the IG and the tenia tecta (Crosby and Schnitzlein, 1982) as part of the olfactory cortex as both receive input from the main olfactory bulb (Wyss and Sripanidkulchai, 1983). In the present study, we confirmed that characteristic histoarchitectonic features are common to the different parts of the IG along its anterior-posterior extent. Moreover, we emphasize that, based on our findings, a continuum between IG and DG can be excluded. Instead, the IG adjoins the hippocampal CA2 region via the FC. However, our study emphasizes that the FC is not identical with the medial CA2 region, as earlier stated (Laeremans et al., 2013). FC and medial CA2 can be distinguished from each other by the perpendicular orientation of their principal cell layers and by differences concerning their developmental protein expression patterns, in particular PCP4 expression. Taking into account our above described experimental findings, we support the interpretation of the IG as an own distinct subregion of the hippocampal formation, in line with a previously proposed definition (Künzle, 2004).

\section{Toward Exploring Functions of the IG}

What could be the functional role of the IG? Although the hippocampal formation is best known for its role in learning and memory and loss of memory in the aging brain (Fjell et al., 2014), in spatial representation and navigating cognition (Bellmund et al., 2018), more recent studies also underpin its involvement in modulating social memory, and particular the CA2 region has been shown to play a crucial role in behavior (Hitti and Siegelbaum, 2014; Dudek et al., 2016; Montagrin et al., 2018; Oliva et al., 2020). While our present study clearly precludes dentate granule cell identity of IG neurons as earlier proposed, we found in contrast that IG neurons express the CA2 markers Necab2 and PCP4. Along this line, IG neurons also express Amigo2 mRNA, which is otherwise specific for CA2 neurons of the hippocampus proper but not expressed in CA1 or CA3 (๔) 2015 Allen Institute for Brain Science. Allen Brain Atlas API. Available from: https://mouse.brain-map.org/experiment/ thumbnails/71250310?image_type=ish\&popup=true; Laeremans et al., 2013; Hitti and Siegelbaum, 2014). Thus, a potential role of the IG in social memory or in other functions related to behavior could be experimentally tested in a similar way as it has been elegantly performed earlier to explore the role of the CA2 region in social memory, i.e., by local inactivation of Amigo2expressing neurons in the CA2 region of Amigo2-Cre mice (Hitti and Siegelbaum, 2014). Next, ex vivo IG explants were recently successfully used for pharmacological treatment of IG principal neurons (Fuzik et al., 2019). Similarly, the use of slice cultures, a well-established in vitro model for hippocampal development and function, may be straightforward to study development, function, and aging of IG neurons in vitro. In particular, IG slice cultures may in the future serve as a novel in vitro model to explore mechanisms that underlie the low vulnerability of IG neurons, notably secretagogin- and PCP4-expressing neurons, in neurodegeneration (Lippa et al., 1990; Lippa and Smith, 1992; Attems et al., 2008). All in all, further investigations on the IG and FC are needed to elucidate specific functions of these distinct hippocampal subregions.

\section{DATA AVAILABILITY STATEMENT}

All datasets generated for this study are included in the article/Supplementary Material, further inquiries can be directed to the corresponding authors.

\section{ETHICS STATEMENT}

The animal study was reviewed and approved by the Landesamt für Natur, Umwelt und Verbraucherschutz Nordrhein-Westfalen, Postfach 101052, 45610 Recklinghausen, Germany.

\section{AUTHOR CONTRIBUTIONS}

$\mathrm{MS}, \mathrm{MvD}$, and $\mathrm{EF}$ designed the study and revised the manuscript. MS and $\mathrm{H}-\mathrm{WH}$ conducted the study and collected the data. MS, MvD, EP-P, and EF analyzed the data, drafted the manuscript, and interpreted the data. All the authors have read and approved the final version of the submitted manuscript.

\section{ACKNOWLEDGMENTS}

We would like to thank Bianca Brunne ${ }^{1}$ and Michael Frotscher ${ }^{1}$, for generously providing eGFP-POMC mice. The excellent technical assistance of Luzie Augustinowski $^{2}$, Sabine Peuckert ${ }^{2}$, Katja Rumpf ${ }^{2}$, Sabine Schreiber-Minjoli ${ }^{2}$, Jeannette Willms ${ }^{2}$, and Corinna Wojczak ${ }^{2}$ is gratefully acknowledged.

${ }^{1}$ Center for Molecular Neurobiology, Hamburg-Eppendorf, Hamburg, Germany.

${ }^{2}$ Department of Neuroanatomy and Molecular Brain Research, Ruhr-University Bochum, Bochum, Germany.

\section{SUPPLEMENTARY MATERIAL}

The Supplementary Material for this article can be found online at: https://www.frontiersin.org/articles/10.3389/fcell.2020. 615571/full\#supplementary-material 


\section{REFERENCES}

Adamek, G. D., Shipley, M. T., and Sanders, M. S. (1984). The indusium griseum in the mouse: architecture, Timm's histochemistry and some afferent connections. Brain Res. Bull. 12, 657-668. doi: 10.1016/0361-9230(84)90147-3

Alpár, A., Attems, J., Mulder, J., Hökfelt, T., and Harkany, T. (2012). The renaissance of $\mathrm{Ca} 2+-$ binding proteins in the nervous system: secretagogin takes center stage. Cell Signal. 24, 378-387. doi: 10.1016/j.cellsig.2011.09.028

Attems, J., Preusser, M., Grosinger-Quass, M., Wagner, L., Lintner, F., and Jellinger, K. (2008). Calcium-binding protein secretagogin-expressing neurones in the human hippocampus are largely resistant to neurodegeneration in Alzheimer's disease. Neuropathol. Appl. Neurobiol. 34, 23-32. doi: 10.1111/j.1365-2990. 2007.00854.x

Bauer, M. C., O'Connell, D. J., Maj, M., Wagner, L., Cahill, D. J., and Linse, S. (2011). Identification of a high-affinity network of secretagogin-binding proteins involved in vesicle secretion. Mol. Biosyst. 7, 2196-2204. doi: 10.1039/ c0mb00349b

Bellmund, J. L. S., Gärdenfors, P., Moser, E. I., and Doeller, C. F. (2018). Navigating cognition: spatial codes for human thinking. Science 362:eaat6766. doi: 10.1126/ science.aat6766

Benadiba, C., Magnani, D., Niquille, M., Morlé, L., Valloton, D., Nawabi, H., et al. (2012). The ciliogenic transcription factor RFX3 regulates early midline distribution of guidepost neurons required for corpus callosum development. PLoS Genet. 8:e1002606. doi: 10.1371/journal.pgen.1002606

Bernier, G., Vukovich, W., Neidhardt, L., Herrmann, B. G., and Gruss, P. (2001). Isolation and characterization of a downstream target of Pax6 in the mammalian retinal primordium. Development 128, 3987-3994.

Bobić Rasonja, M., Orešković, D., Knezović, V., Pogledić, I., Pupačić, D., Vukšić, M., et al. (2019). Histological and MRI study of the development of the human indusium griseum. Cereb. Cortex 29, 4709-4724. doi: 10.1093/cercor/ bhz004

Cabin, D. E., Gardiner, K., and Reeves, R. H. (1996). Molecular genetic characterization and comparative mapping of the human PCP4 gene. Somat. Cell Mol. Genet. 22, 167-175. doi: 10.1007/BF02369907

Celio, M. R. (1990). Calbindin D-28k and parvalbumin in the rat nervous system. Neuroscience 35, 375-475. doi: 10.1016/0306-4522(90) 90091-h

Chen, H., Bouras, C., and Antonarakis, S. E. (1996). Cloning of the cDNA for a human homolog of the rat PEP-19 gene and mapping to chromosome 21q22.2-q22.3. Hum. Genet. 98, 672-677. doi: 10.1007/s004390050282

Crosby, E. C., and Schnitzlein, H. N. (1982). Comparative Correlative Neuroanatomy of the Vertebrate Telencephalon. New York, NY: Macmillan.

Di Ieva, A., Fathalla, H., Cusimano, M. D., and Tschabitscher, M. (2015). The indusium griseum and the longitudinal striae of the corpus callosum. Cortex 62, 34-40. doi: 10.1016/j.cortex.2014.06.016

Dudek, S. M., Alexander, G. M., and Farris, S. (2016). Rediscovering area CA2: unique properties and functions. Nat. Rev. Neurosci. 17, 89-102. doi: 10.1038/ nrn.2015.22

Elliot Smith, G. (1897). The morphology of the indusium and striae lancisii. Anat. Anz. 13, 23-27.

Fjell, A. M., McEvoy, L., Holland, D., Dale, A. M., and Walhovd, K. B. (2014). What is normal in normal aging? Effects of aging, amyloid and Alzheimer's disease on the cerebral cortex and the hippocampus. Prog. Neurobiol. 117, 20-40. doi: 10.1016/j.pneurobio.2014.02.004

Fuzik, J., Rehman, S., Girach, F., Miklosi, A. G., Korchynska, S., Arque, G., et al. (2019). Brain-wide genetic mapping identifies the indusium griseum as a prenatal target of pharmacologically unrelated psychostimulants. Proc. Natl. Acad. Sci. U.S.A. 116, 25958-25967. doi: 10.1073/pnas.1904 006116

Harashima, S.-I., Wang, Y., Horiuchi, T., Seino, Y., and Inagaki, N. (2011). Purkinje cell protein 4 positively regulates neurite outgrowth and neurotransmitter release. J. Neurosci. Res. 89, 1519-1530. doi: 10.1002/jnr.22688

Hitti, F. L., and Siegelbaum, S. A. (2014). The hippocampal CA2 region is essential for social memory. Nature 508, 88-92. doi: 10.1038/nature13028

Ishizawa, T., Ko, L.-W., Cookson, N., Davias, P., Espinoza, M., and Dickson, D. W. (2002). Selective neurofibrillary degeneration of the hippocampal CA2 sector is associated with four-repeat tauopathies. J. Neuropathol. Exp. Neurol. 61, 1040-1047. doi: 10.1093/jnen/61.12.1040
Iwamoto, K., Bundo, M., Yamamoto, M., Ozawa, H., Saito, T., and Kato, T. (2004). Decreased expression of NEFH and PCP4/PEP19 in the prefrontal cortex of alcoholics. Neurosci. Res. 49, 379-385. doi: 10.1016/j.neures.2004.04.002

Iwano, T., Masuda, A., Kiyonari, H., Enomoto, H., and Matsuzaki, F. (2012). Prox1 postmitotically defines dentate gyrus cells by specifying granule cell identity over CA3 pyramidal cell fate in the hippocampus. Development 139, 3051-3062. doi: $10.1242 / \mathrm{dev} .080002$

Jacobowitz, D. M., and Winsky, L. (1991). Immunocytochemical localization of calretinin in the forebrain of the rat. J. Comp. Neurol. 304, 198-218. doi: 10. 1002/cne.903040205

Kjonigsen, L. J., Leergaard, T. B., Witter, M. P., and Bjaalie, J. G. (2011). Digital atlas of anatomical subdivisions and boundaries of the rat hippocampal region. Front. Neuroinform. 5:2. doi: 10.3389/fninf.2011. 00002

Künzle, H. (2004). The hippocampal continuation (indusium griseum): its connectivity in the hedgehog tenrec and its status within the hippocampal formation of higher vertebrates. Anat. Embryol. 208, 183-213. doi: 10.1007/ s00429-004-0384-3

Laeremans, A., Nys, J., Luyten, W., D’Hooge, R., Paulussen, M., and Arckens, L. (2013). AMIGO2 mRNA expression in hippocampal CA2 and CA3a. Brain Struct. Funct. 218, 123-130. doi: 10.1007/s00429-012-0387-4

Laplante, F., Mnie-Filali, O., and Sullivan, R. M. (2013). A neuroanatomical and neurochemical study of the indusium griseum and anterior hippocampal continuation: comparison with dentate gyrus. J. Chem. Neuroanat. 5, 39-47. doi: 10.1016/j.jchemneu.2013.03.004

Lavado, A., Lagutin, O. V., Chow, L. M. L., Baker, S. J., and Oliver, G. (2010). Prox1 is required for granule cell maturation and intermediate progenitor maintenance during brain neurogenesis. PLoS Biol. 8:e1000460. doi: 10.1371/ journal.pbio. 1000460

Lavado, A., and Oliver, G. (2007). Prox1 expression patterns in the developing and adult murine brain. Dev. Dyn. 236, 518-524. doi: 10.1002/dvdy.21024

Lavado, A., Ware, M., Paré, J., and Cao, X. (2014). The tumor suppressor $\mathrm{Nf} 2$ regulates corpus callosum development by inhibiting the transcriptional coactivator Yap. Development 141, 4182-4193. doi: 10.1242/dev.111260

Lein, E. S., Callaway, E. M., Albright, T. D., and Gage, F. H. (2005). Redefining the boundaries of the hippocampal CA2 subfield in the mouse using gene expression and 3-dimensional reconstruction. J. Comp. Neurol. 485, 1-10. doi: $10.1002 /$ cne. 20426

Lippa, C. F., and Smith, T. W. (1992). The indusium griseum in Alzheimer's disease: an immunocytochemical study. J. Neurol. Sci. 111, 39-45. doi: 10.1016/0022510x(92)90110-7

Lippa, C. F., Smith, T. W., Degirolami, U., and Drachman, D. A. (1990). The indusium griseum: is it involved in Alzheimer's disease? Neurobiol. Aging 11, 551-554. doi: 10.1016/0197-4580(90)90116-h

Macchi, G. (1951). The ontogenetic development of the olfactory telencephalon in man. J. Comp. Neurol. 95, 245-305. doi: 10.1002/cne.900950203

Maj, M., Gartner, W., Ilhan, A., Neziri, D., Attems, J., and Wagner, L. (2010). Expression of TAU in insulin-secreting cells and its interaction with the calcium-binding protein secretagogin. J. Endocrinol. 205, 25-36. doi: 10.1677/ JOE-09-0341

Maj, M., Milenkovic, I., Bauer, J., Berggård, T., Veit, M., Ilhan-Mutlu, A., et al. (2012). Novel insights into the distribution and functional aspects of the calcium binding protein secretagogin from studies on rat brain and primary neuronal cell culture. Front. Mol. Neurosci. 5:84. doi: 10.3389/fnmol.2012. 00084

Montagrin, A., Saiote, C., and Schiller, D. (2018). The social hippocampus. Hippocampus 28, 672-679. doi: 10.1002/hipo.22797

Mulder, J., Spence, L., Tortoriello, G., Dinieri, J. A., Uhlén, M., Shui, B., et al. (2010). Secretagogin is a Ca2+-binding protein identifying prospective extended amygdala neurons in the developing mammalian telencephalon. Eur. J. Neurosci. 31, 2166-2177. doi: 10.1111/j.1460-9568.2010.07275.x

Mulder, J., Zilberter, M., Spence, L., Tortoriello, G., Uhlén, M., Yanagawa, Y., et al. (2009). Secretagogin is a Ca2+-binding protein specifying subpopulations of telencephalic neurons. Proc. Natl. Acad. Sci. U.S.A. 106, 22492-22497. doi: 10.1073/pnas.0912484106

Oliva, A., Fernández-Ruiz, A., Leroy, F., and Siegelbaum, S. A. (2020). Hippocampal CA2 sharp-wave ripples reactivate and promote social memory. Nature 587, 264-269. doi: 10.1038/s41586-020-2758-y 
Oliver, G., Sosa-Pineda, B., Geisendorf, S., Spana, E. P., Doe, C. Q., and Gruss, P. (1993). Prox 1, a prospero-related homeobox gene expressed during mouse development. Mech. Dev. 44, 3-16. doi: 10.1016/0925-4773(93) 90012-m

Overstreet, L. S., Hentges, S. T., Bumaschny, V. F., de Souza Flavio, S. J., Smart, J. L., and Santangelo, A. M. (2004). A transgenic marker for newly born granule cells in dentate gyrus. J. Neurosci. 24, 3251-3259. doi: 10.1523/JNEUROSCI.517303.2004

Paxinos, G., and Franklin, K. B. J. (2019). Paxinos and Franklin's the Mouse Brain in Stereotaxic Coordinates. Cambridge, MA: Academic Press.

Paxinos, G., and Watson, C. (2009). Chemoarchitectonic Atlas of the Mouse Brain. Cambridge, MA: Academic Press.

Petrasch-Parwez, E., Nguyen, H.-P., Löbbecke-Schumacher, M., Habbes, H.-W., Wieczorek, S., Riess, O., et al. (2007). Cellular and subcellular localization of Huntingtin corrected aggregates in the brain of a rat transgenic for Huntington disease. J. Comp. Neurol. 501, 716-730. doi: 10.1002/cne.21272

Recabarren, D., and Alarcón, M. (2017). Gene networks in neurodegenerative disorders. Life Sci. 183, 83-97. doi: 10.1016/j.lfs.2017.06.009

Renelt, M., von Bohlen und Halbach, V., and von Bohlen und Halbach, O. (2014). Distribution of PCP4 protein in the forebrain of adult mice. Acta Histochem. 116, 1056-1061. doi: 10.1016/j.acthis.2014.04.012

San Antonio, A., Liban, K., Ikrar, T., Tsyganovskiy, E., and Xu, X. (2014). Distinct physiological and developmental properties of hippocampal CA2 subfield revealed by using anti-Purkinje cell protein 4 (PCP4) immunostaining. J. Comp. Neurol. 522, 1333-1354. doi: 10.1002/cne.23486

Shu, T., Puche, A. C., and Richards, L. J. (2003). Development of midline glial populations at the corticoseptal boundary. J. Neurobiol. 57, 81-94. doi: 10.1002/ neu. 10252

Slemmon, J. R., Hughes, C. M., Campbell, G. A., and Flood, D. G. (1994). Increased levels of hemoglobin-derived and other peptides in Alzheimer's disease cerebellum. J. Neurosci. 14, 2225-2235. doi: 10.1523/JNEUROSCI.1404-02225.1994

Smith, K. M., Ohkubo, Y., Maragnoli, M. E., Rasin, M.-R., Schwartz, M. L., Sestan, N., et al. (2006). Midline radial glia translocation and corpus callosum formation require FGF signaling. Nat. Neurosci. 9, 787-797. doi: 10.1038/ nn1705

Sugita, S., Ho, A., and Südhof, T. C. (2002). NECABs: a family of neuronal $\mathrm{Ca}(2+)$-binding proteins with an unusual domain structure and a restricted expression pattern. Neuroscience 112, 51-63. doi: 10.1016/s0306-4522(02) 00063-5

Teyssier, J.-R., Ragot, S., Chauvet-Gélinier, J.-C., Trojak, B., and Bonin, B. (2011). Activation of a $\triangle F O S B$ dependent gene expression pattern in the dorsolateral prefrontal cortex of patients with major depressive disorder. J. Affect. Disord. 133, 174-178. doi: 10.1016/j.jad.2011.04.021

Utal, A. K., Stopka, A. L., Roy, M., and Coleman, P. D. (1998). PEP19 immunohistochemistry defines the basal ganglia and associated structures in the adult human brain, and is dramatically reduced in Huntington's disease. Neuroscience 86, 1055-1063. doi: 10.1016/s0306-4522(98) 00130-4

Wyss, J. M., and Sripanidkulchai, K. (1983). The indusium griseum and anterior hippocampal continuation in the rat. J. Comp. Neurol. 219, 251-272. doi: 10. 1002/cne.902190302

Zahola, P., Hanics, J., Pintér, A., Máté, Z., Gáspárdy, A., Hevesi, Z., et al. (2019). Secretagogin expression in the vertebrate brainstem with focus on the noradrenergic system and implications for Alzheimer's disease. Brain Struct. Funct. 224, 2061-2078. doi: 10.1007/s00429-019-01886-w

Zhang, M.-D., Su, J., Adori, C., Cinquina, V., Malenczyk, K., Girach, F., et al. (2018). Ca2+-binding protein NECAB2 facilitates inflammatory pain hypersensitivity. J. Clin. Invest. 128, 3757-3768. doi: 10.1172/JCI1 20913

Ziai, M. R., Sangameswaran, L., Hempstead, J. L., Danho, W., and Morgan, J. I. (1988). An immunochemical analysis of the distribution of a brain-specific polypeptide, PEP-19. J. Neurochem. 51, 1771-1776. doi: 10.1111/j.1471-4159. 1988.tb01158.x

Ziai, R., Pan, Y. C., Hulmes, J. D., Sangameswaran, L., and Morgan, J. I. (1986). Isolation, sequence, and developmental profile of a brain-specific polypeptide, PEP-19. Proc. Natl. Acad. Sci. U.S.A. 83, 8420-8423. doi: 10.1073/pnas.83.21. 8420

Zimmermann, B., Girard, F., Mészàr, Z., and Celio, M. R. (2013). Expression of the calcium binding proteins Necab-1,-2 and -3 in the adult mouse hippocampus and dentate gyrus. Brain Res. 1528, 1-7. doi: 10.1016/j.brainres.2013. 06.004

Conflict of Interest: The authors declare that the research was conducted in the absence of any commercial or financial relationships that could be construed as a potential conflict of interest.

Copyright (c) 2021 Sanders, Petrasch-Parwez, Habbes, Düring and Förster. This is an open-access article distributed under the terms of the Creative Commons Attribution License (CC BY). The use, distribution or reproduction in other forums is permitted, provided the original author(s) and the copyright owner(s) are credited and that the original publication in this journal is cited, in accordance with accepted academic practice. No use, distribution or reproduction is permitted which does not comply with these terms. 